Article

\title{
Tuning Nanoscale Friction by Applying Weak Magnetic Fields to Reorient Adsorbed Oxygen Molecules
}

\author{
Z. B. Fredricks, K. M. Stevens, S. G. Kenny, B. Acharya ${ }^{\mathbb{D}}$ and J. Krim *
}

Department of Physics, North Carolina State University, Raleigh, NC 27695-7218, USA; zfredri@ncsu.edu (Z.B.F.); keeley@gmail.com (K.M.S.); sgkenny@ncsu.edu (S.G.K.); bachary@ncsu.edu (B.A.)

* Correspondence: jkrim@ncsu.edu

Received: 14 November 2018; Accepted: 18 December 2018; Published: 20 December 2018

\begin{abstract}
Sliding friction levels of thin (1-2 monolayers) and thick ( 10 monolayers) oxygen films adsorbed on nickel and gold at $47.5 \mathrm{~K}$ have been measured by means of a quartz crystal microbalance (QCM) technique. Friction levels for the thin (thick) films on nickel in the presence of a weak magnetic field were observed to be approximately $30 \%(50 \%)$ lower than those recorded in the absence of the external field. Friction levels for thin films on gold were meanwhile observed to be substantially increased in the presence of the field. Magnetically-induced structural reorientation (magnetostriction) and/or realignment of adlayer spins, which respectively reduce structural and magnetic interfacial corrugation and commensurability, appear likely mechanisms underlying the observed field-induced reductions in friction for the nickel samples. Eddy current formation in the gold substrates may account for the increased friction levels in this system. The work demonstrates the role of magnetic effects in model systems that are highly amenable to theoretical studies and modeling.
\end{abstract}

Keywords: tribology; magnetic friction; atomic-scale friction; oxygen; nickel; gold; quartz crystal microbalance

\section{Introduction}

The molecular origins of friction, an important physical phenomenon in light of both its everyday familiarity and its enormous economic impact [1], have been discussed and debated for hundreds of years. The topic has accelerated in recent decades [2,3], spurred by the discovery and documentation of fundamental mechanisms for frictional energy dissipation in the complete absence of wear [4], including phonons [4], ohmic damping [5,6], the creation of electron-hole pairs, and/or electrostatic effects $[7,8]$. Magnetic friction, the topic of the present study, has been explored to a far lesser extent [6]. Recent studies have concluded, however, that in addition to phononic and electronic effects, spin dissipation mechanisms can be significant for systems involving magnetic materials, including at the macroscopic scale [9-12]. Adatom diffusivity, a manifestation of friction, has been linked to the magnetic properties of adsorbate-substrate systems [13,14], and adatoms can also be used to amplify probes of surface spin texture [15].

Magnetism can impact friction in a variety of ways, from a trivial reduction in contact area associated with magnetic levitation [16], to intrinsically magnetic dissipative mechanisms such as spin waves $[17,18]$ and/or spin flips $[11,12,19]$, excited by interfacial lateral motion. The transient resistive forces associated with conduction electron eddy currents arising from relative motion of a magnetic material with respect to an electrical conductor [20] are routinely referred to as "magnetic friction", but are more correctly categorized as a manifestation of conduction electron dissipative effects. Magnetic fields can also influence the physical structure of materials, resulting in secondary effects that 
impact friction. These include magnetostrictive shape changes in solids [21-23], as well as viscosity and magnetorheological stiffness changes in liquids [24], which alter interfacial commensurability and, thus, the phononic contributions to friction [2-4].

Experimental studies targeting magnetic dissipative mechanisms to date have been very limited in number, and have employed asperity-substrate geometries in either contact [12] or non-contact geometries [19]. Although planar interfacial geometries remain virtually unexplored, a variety of fascinating dissipative phenomena have been theoretically predicted, in association with varying degrees of magnetic commensurability and/or interfacial spin corrugation [11]. Kadau et al., for example, studied the intrinsic magnetic interaction between two magnetic planes of magnetic materials, both of which were insulating so as to avoid conduction electron and eddy current effects. One major finding of the study was that that anti-ferromagnetic interfaces resulted in significantly higher friction levels than ferromagnetic interfaces. Planar geometries are also amenable to modeling of the magnetic and electronic properties of a wide range of magnetic materials. For the present study, we employed oxygen films, which are paramagnetic, adsorbed in a planar geometry on ferromagnetic nickel substrates and nonmagnetic gold substrates in the presence and absence of weak external magnetic fields, to explore whether the fields could be used to actively tune adsorbate friction levels.

Numerous experimental and theoretical studies have confirmed magnetically ordered monolayers for the solid phases of adsorbed oxygen, including an antiferromagnetic phase $[25,26]$. When exposed to an external field, such layers, when adsorbed on graphite, are reported to exhibit behavior more characteristic of a ferromagnet than a paramagnet, achieving saturated magnetism even in the presence of weak external magnetic fields whose field energy is small compared with the thermal energy of the molecules [27]. The effect is expected to be present for gold substrates as well, and enhanced for adsorption on nickel substrates. The energetically favorable, or "easy" direction of spontaneous magnetization of adsorbed films comprised of upright molecules is along the $z$-axis of the molecules, in contrast to the spin direction of isolated molecules, which is perpendicular to the $z$-axis.

The experiment geometry is depicted schematically in Figure 1. In part (a) adsorbed oxygen molecules slide on the substrate in the absence of an externally applied field. The physical orientation of the molecules, as well as their spin direction, is determined by the combined effects of molecule-molecule and molecule-substrate interactions, which are van der Waals in nature. When an external field is applied (Figure 1b), the attractive interaction between the paramagnetic moments of the adlayer and the ferromagnetic moments of the substrate will act to align the molecules and also increase attraction with the substrate. Increased attraction does not increase contact area, as it would in a macroscopic system, but a change in friction arising from a change in the effective surface corrugation is to be expected. Alignment of the oxygen molecules in response to the external field [22] would in addition change the commensurability with the substrate, reducing friction if the molecules become more incommensurate and solid-like $[2,4,28]$. Finally, the presence of the external field could also decrease the magnetic corrugation of the interface, if the oxygen layer spins reorient from a preferentially anti-ferromagnetic to preferentially ferromagnetic state. This would reduce friction associated with spin-waves and spin-flips [11]. The sliding oscillatory motion of magnetic adlayers would also be expected to give rise to eddy currents in the underlying substrate [20] and cause friction levels to increase.

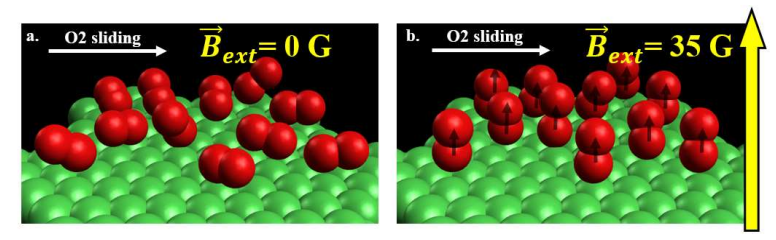

Figure 1. Illustration of the effect of a magnetic field onto physisorbed $\mathrm{O}_{2}$ films. When dosed into the system with the absence of external field. (a) the molecules are unordered, in physical orientation and spin direction, with respect to the surface. In contrast, when adsorbed in the presence of a weak magnetic field oriented perpendicular to the substrate. (b) the molecules can align, both in physical aspect and in spin moment, with respect to the field. This reduces the magnetic frictional interaction of sliding films. 


\section{Experimental}

Measurements were recorded by means of a quartz crystal microbalance (QCM) at $47 \mathrm{~K}$, where oxygen molecules are physisorbed to the substrate and known to remain in a magnetic state upon uptake on the surface [25-36]. At $47 \mathrm{~K}$, adsorbed oxygen layers are liquid, but close to their two-dimensional solid-liquid monolayer melting point, and have structural orientation imposed by the substrate. Molecules initially adsorb in a direction preferentially parallel to the substrate, then progressively tilt upward as coverage increases. The behavior, which is very general for physisorbed oxygen monolayers, has been reported for graphite [22,25-32] and $\mathrm{Ni}(111)$ substrates coated with disassociated oxygen atoms [33-36]. Above monolayer coverage, a surface melted second layer forms atop the monolayer that is incommensurate with the layer adjacent to the substrate $[29,30]$.

The quartz crystal microbalance (QCM) is an established technique for measuring friction levels in adsorbed films [37-39]. Film uptake on QCM surface electrodes produces a shift in the frequency Sf associated with the inertial mass tracking the horizontal oscillatory motion of the QCM [40], and quality factor shifts associated with energy dissipation arising from frictional sliding motion of film constituents [40,41]. The ratio $\delta(1 / Q) /(\delta f)$ is reflective of the form of frictional shear forces with the substrate. For adsorbed films it is routinely parameterized by:

$$
\delta(1 / Q) /(\delta \mathrm{f})=4 \pi \tau
$$

where $\tau$ is a characteristic slip time associated the time decay of momentum fluctuations in the film. The form of the friction law can be probed by studies of how the slip time $\tau$ varies with the QCM's amplitude of vibration $A$ according to:

$$
F=-\gamma v^{n} \operatorname{sgn}(v), \delta(1 / \mathrm{Q}) \propto \tau \cong A^{-(1-1 / n)}
$$

where $\gamma$ is a constant and $v$ is the relative sliding speed. The constant [42] $\mathrm{n}=0$ corresponds to the common macroscopic friction law $\mathrm{F}=\mu \mathrm{N}, \mathrm{n}=1$ corresponds to linear viscous friction, and $\mathrm{n}>1$ is commonly associated with granular materials [43]. Various first principle theoretical and numerical studies have found the $\mathrm{n}=1$ case of linear friction to apply to adsorbed films, so long as the sliding distance is more than a few atomic lattice spacings and sliding speeds remain below the speed of sound, conditions that apply to the present study [44-48]. Particularly notable in these studies is the fact that exceptionally small changes in commensurability between adsorbed film particles and the substrate can result in many orders of magnitude in the friction, a feature that will be discussed later within the context of the data presented.

A QCM's measured frequency shift is related to the shift $\delta f_{\text {film }}$ of a rigidly adhering film according to:

$$
\delta f\left[1+(\omega \tau)^{2}\right]=\delta f_{\text {film }}
$$

Therefore studies of adsorbed layers are generally performed with QCM's operating in the fundamental mode, as sensitivity and activation areas become problematic with modes for systems that exhibit significant slippage $[49,50]$.

The QCMs employed here consisted of single crystal overtone-polished AT(A-type Transverse shear mode)-cut quartz obtained from Laptech Precision Instruments (Bowmanville, ON, Canada) (part number XL1018) that oscillated in transverse shear motion with a quality factor close to $10^{5}$ and driven by a Pierce oscillator circuit at the series resonance frequency $8.01 \mathrm{MHz}$ of the oscillator (Figure 2). For the gold substrates, the crystals were coated with Au by the manufacturer and employed as prepared. For the nickel substrates, the QCM's were obtained from the same manufacturer, but without electrodes. The blank crystals were mounted within a masked holder and baked in air at $150{ }^{\circ} \mathrm{C}$ for $24 \mathrm{~h}$ before insertion in an ultra-high vacuum deposition chamber (base pressure $10^{-9}-10^{-10}$ Torr) where nickel electrodes were deposited. Thirty-nanometer thick Ni electrodes were deposited onto each of the two sides of the QCM at a rate of $0.1 \mathrm{~nm} / \mathrm{min}$ by means of a tungsten filament e-beam gun targeted upon a 
$\mathrm{Ni}$ rod held at $4.2 \mathrm{kV}$. After deposition of the electrodes, the sample was annealed at $250{ }^{\circ} \mathrm{C}$ for several hours, then transferred in air to a separate cryogenic stage within a vacuum chamber comprised of ultra-high vacuum components, then baked overnight at $140^{\circ} \mathrm{C}$ at $10^{-8}$ Torr to desorb physisorbed contaminants. Nickel films prepared in this manner exhibit a preferred (111) orientation [51-53]. Residual disassociated oxygen atoms chemisorbed on the Ni sample in the course of its exposure to air are reported to be ferromagnetic in nature [34].

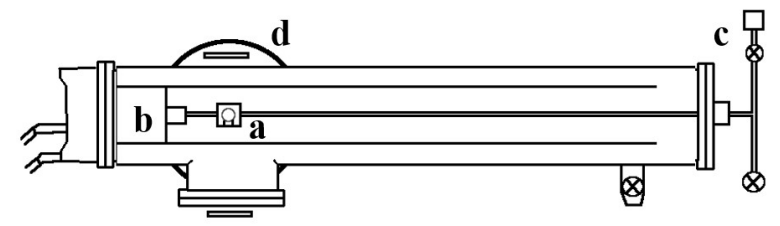

Figure 2. UHV vacuum chamber with QCM sample (a) held at $47 \mathrm{~K}$ connected to $4 \mathrm{~K}$ cold tip (b). The sample is dosed with a fixed amount of $\mathrm{O}_{2}$ gas from (c). External magnetic field is applied via an electromagnet $(\mathbf{d})$.

Both gold and nickel samples were cooled to $47 \mathrm{~K}$ by means of a Sumitomo cryocooler (Allentown, PA, USA) with temperature controlled by means of a Lakeshore (Westerville, OH, USA) controller and silicon diode thermometer. Data for frequency and quality factor shifts were recorded for film coverages of approximately 70 and $200 \mathrm{ng} / \mathrm{cm}^{2}$, by dosing two distinct fixed volumes of research grade $\mathrm{O}_{2}$ slowly into the chamber containing the QCM sample held at low temperature. A Labview program (National Instruments, Austin, TX, USA) continuously recorded the temperature, frequency and amplitude data every five seconds during dosage and as the samples equilibrated. The procedure was repeated for numerous runs in both the presence and absence of a $35 \mathrm{G}$ magnetic field applied in a directly perpendicular to the surface. Frequency and quality factor shifts associated with residual gas pressure, tensile stress, and/or temperature variations were negligible for the experimental conditions employed for the present studies.

The magnetic field was produced by means of a GMW 5451 electromagnet (San Carlos, CA, USA) with field homogeneity of 200 ppm in the central region, in which the sample cube resides, and calibrated with a Lakeshore 425 gaussmeter. It was held constant during all data runs.

The sample cell consisted of a ultra-high-vacuum compatible six-way cube attached to the cold tip by means of a custom machined oxygen free high thermal conductivity (OFHC) heat bridge of diameter $3 / 8^{\prime \prime}$. The typical base pressure within the cube, accounting for thermal transpiration, was estimated to be $7 \times 10^{-10}$ Torr. Both tip side and sample side are mounted resistance thermometers (RTDs) for temperature measurement. Typical thermal gradients exist across the bridge 5-10 K, and at steady state $47 \mathrm{~K}$, this thermal gradient is about $3 \mathrm{~K}$. Since the temperature gradient at steady state between the RTDs is small, we can expect the sample temperature to be trivially close to the sample RTD's reading at steady state.

Dosage of $\mathrm{O}_{2}$ onto the sample surface was performed conducted at low temperature after thermal stability $>0.01 \mathrm{~K}$ had been established. Oxygen was deposited by means of a vacuum leak-in valve, behind which a fixed volume containing $7.0 \mathrm{~m} \cdot$ Torr $\mathrm{O}_{2}$ was present. After opening the valve to admit a fixed dose of gas, the pressure dropped to $2 \mathrm{~m}$. Torr.

Great care was taken to perform control studies to examine whether a temperature change occurred when the gas was admitted, and we observed that the room temperature gas quickly established equilibrium and a stronger thermal link between the sample and cold point, cooling the sample further by $0.049 \pm 0.003 \mathrm{~K}$. For the higher dosage runs, the cooling effect saturates to $0.39 \pm 0.03 \mathrm{~K}$ at a $47.5 \mathrm{~K}$ setpoint. We observed no thermal annealing effects such as those reported in experimental setups employed in independent studies [54] and no effects of the dosing rate on the equilibrium times. The films were observed to reach equilibrium typically within $15-20 \mathrm{~min}$, comparable to that reported for independent setups. [55] 


\section{Results}

\subsection{Oxygen Films on Nickel}

Figure 3a-d display characteristic raw data sets for the four conditions studied. Filled circles denote the presence of the magnetic field while stars correspond to zero field conditions. Plots $3 a$ and $3 \mathrm{~b}$ display thick film deposition, while $3 \mathrm{c}$ and $3 \mathrm{~d}$ display monolayer thin film. Plots $3 \mathrm{e}$ and $3 \mathrm{f}$ depict the QCM response in empty cell when the external magnetic is switched 'on', showing the level of stability and null response of system. The horizontal dashed lines in Figure $3 a-d$ are for visual guide, to highlight the differences in the observed data sets, particularly in the inverse quality factor $\delta(1 / Q)$ shifts in the present and absence of an external field.
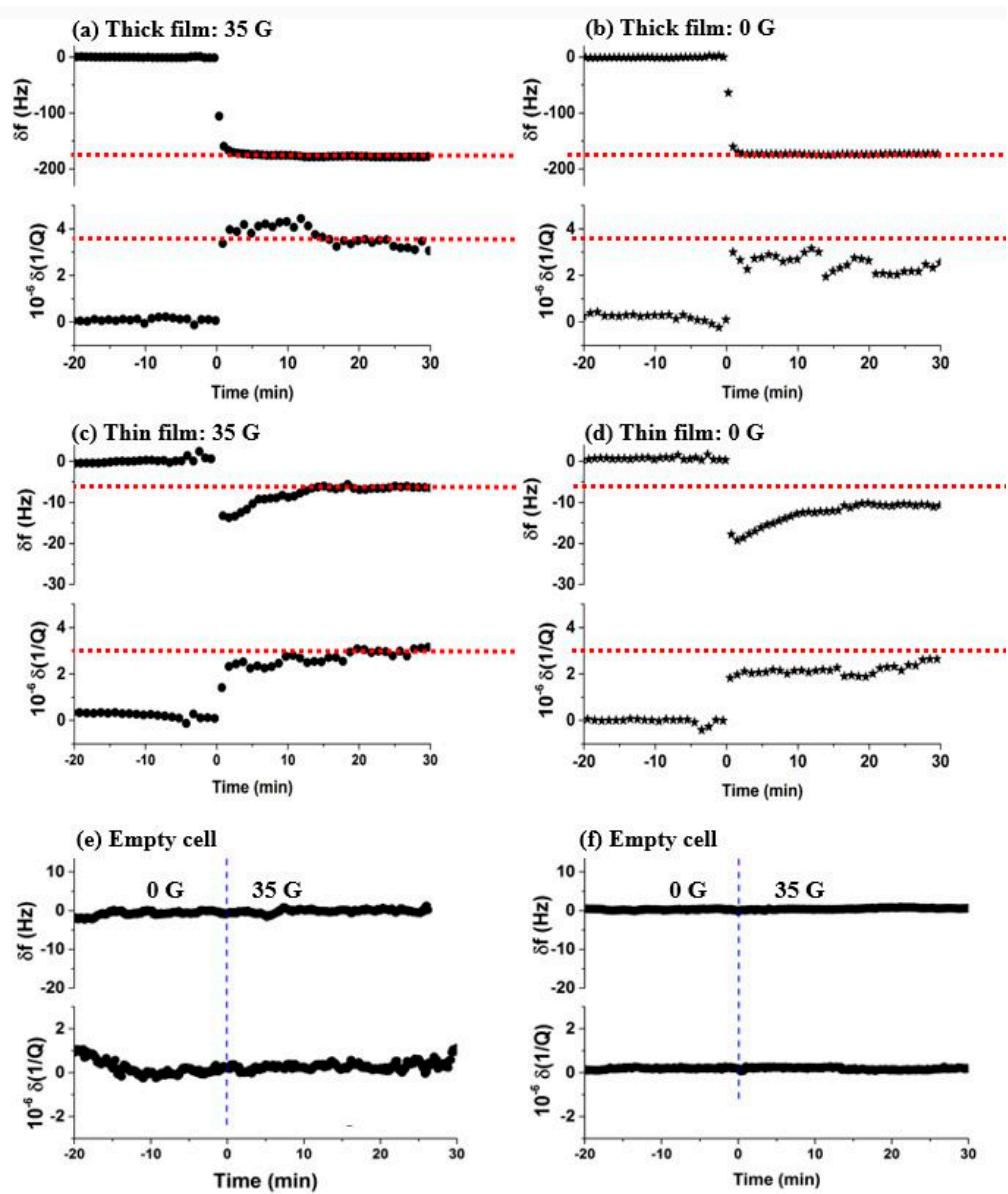

Figure 3. (a-d) Frequency and inverse quality factor response to uptake of thin and thick $\mathrm{O}_{2}$ films deposited $(a t t=0)$ on nickel in the presence and absence of field. Filled circles correspond to field $(\mathbf{a}, \mathbf{c})$, and stars correspond to the field absent case $(\mathbf{b}, \mathbf{d})$. $(\mathbf{a}, \mathbf{b})$ correspond to thick film deposited using relatively large dosage of oxygen whereas $(\mathbf{c}, \mathbf{d})$ correspond to thin film. The horizontal dashed lines $(\mathbf{a}-\mathbf{d})$ are for visual guide, and represent the levels of frequency shift $\delta \mathrm{f}$ and shift in inverse quality factor $\delta(1 / Q)$ for the thick and thin films deposited in the presence of field. $(\mathbf{e}, \mathbf{f})$ represent the system response in empty cell when the magnetic field is switched 'on' $(t=0)$, showing no effect to the QCM responses in the absence of gas. For thin film in the presence of field (c), $\delta \mathrm{f}$ is smaller and $\delta(1 / \mathrm{Q})$ is larger than their corresponding values in the absence of field (d). Therefore, the slip time $\tau$ (Equation 1) for the thin film is larger implying lower friction in the field. Similarly, lower friction in the field is demonstrated for the thick film by the larger resultant quality factor shift although the frequency shifts are comparable in two cases $(\mathbf{a}, \mathbf{b})$. 
One monolayer of oxygen standing entirely upright has coverage 11.45 molecules $/ \mathrm{nm}^{2}$, or $59.9 \mathrm{ng} / \mathrm{cm}^{2}$, which corresponds to a $16.4 \mathrm{~Hz}$ drop in frequency for adsorption on an $8.01 \mathrm{MHz}$ QCM. One monolayer of adsorbed liquid oxygen meanwhile has coverage 7.63 molecules $/ \mathrm{nm}^{2}$, corresponding to $40.6 \mathrm{ng} / \mathrm{cm}^{2}$, or a $10.9 \mathrm{~Hz}$ drop in frequency. The frequency shifts observed for the case of thick films are observed to be close to ten times greater than the thin film case. Shifts in inverse quality factor are observed to be slightly larger when the field is present. Thin films exhibited frequency shifts in the range $15-20 \mathrm{~Hz}$, corresponding to slightly above monolayer coverage, and more pronounced differences in the inverse quality factor shifts in the presence of an external field. While the frequency shifts for the thick film in the presence and absence of field are close, the shift in inverse quality factor for the former is higher than the latter case. The higher shifts in inverse quality factor in the field for both thin and thick film implied the larger slip times and lower friction levels compared to no field cases. When averaged over multiple runs, such differences results in statistically distinct friction levels for the various conditions studied.

Figure 4 displays the results for the slip time analysis for multiple runs for the thick and thin films. Slip time averaged over multiple data sets as a function of time post $\mathrm{O}_{2}$ deposition is plotted for both thin and thick films and field present and absent cases. The slip times observed here range from $1-20 \mathrm{~ns}$, corresponding to $\omega \tau=0.05-1.00$, and "mass sensitivity factors", $\delta \mathrm{f} / \delta \mathrm{f}_{\text {film }}=1 /\left(1+(\omega \tau)^{2}\right)$ that range from 0.997 to 0.497 . For the lowest friction values, the mass per unit area of the adsorbed film is therefore double of that inferred from the frequency shift without corrections. For thin films, the graphs in both the field absent and present cases, ca. Figure $4 a$, have similar nature that the slip time increase monotonically and asymptote to steady state after about $30 \mathrm{~min}$. Additionally, the frequency shift appears to depend on presence of the field for thin films, ca. Figure 3c,d. This is however attributable to apparent coverage being reduced due to larger slip time value close to $20 \mathrm{~ns}$, and therefore a reduced coupling of the substrate with the adsorbed film. The thin film corrected coverages, for the field present and field absent cases are very similar, on the order of $70 \mathrm{ng} / \mathrm{cm}^{2}$, when Equation 2 is utilized to determine the frequency shift corresponding to the actual film coverage.

For the thick film case in Figure $4 \mathrm{~b}$, the increase in slip time is observed in the presence of field, however the average values in two cases fall within the error bars as the time progress. A higher level of external field is expected to enhance the effect. However, the Ni substrates being magnetic, the field level is limited to $35 \mathrm{G}$ for the experiments reported herein. Further studies with higher external field will be essential to confirm this claim. The proportional increase in slip time due to the field is weaker for thick film than the thin film, for which, an increase from $8 \mathrm{~ns}$ to $13 \mathrm{~ns}$ is observed. As compared to the thin film case, the slip time increase in thick film is much smaller, about a factor of ten. The evolution of slip time over time is flatter for the thick film case as compared to the thin film, implying the thick films establish a frictional equilibrium at a faster rate than thin films.
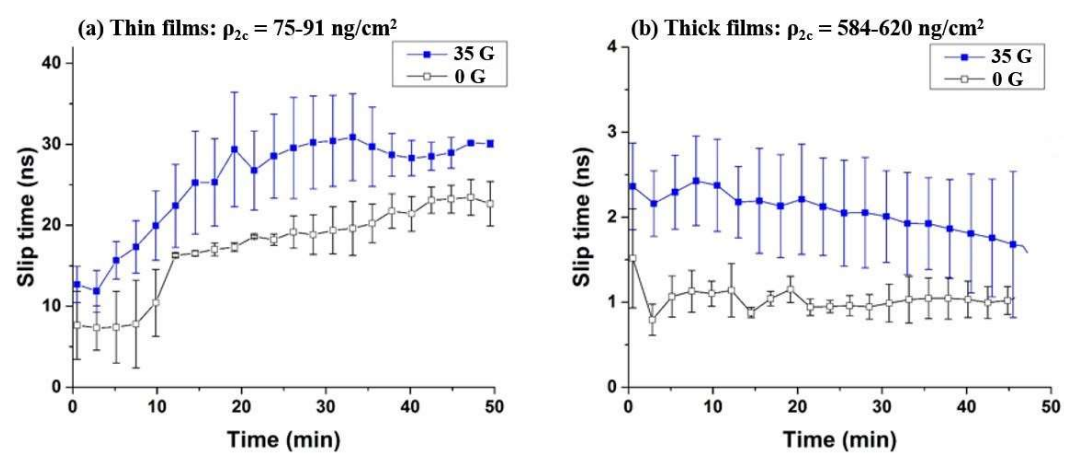

Figure 4. Average slip time versus time for thin (a) and thick (b) oxygen films on nickel in the presence and absence of an external magnetic field perpendicular to the surfaces. Error bars represent the standard deviation of the slip time values averaged over the various runs at fixed points in time. Gas is admitted to the chamber at time $=0 \mathrm{~min}$. Slip times are observed to be longer in the presence of an external magnetic field of $35 \mathrm{G}$, indicating lower friction levels. 


\subsection{Oxygen Films on Gold}

Figure 5 presents slip time data for thin oxygen films on gold for field levels of 0,35 and $70 \mathrm{G}$. For the nickel films studied, the $35 \mathrm{G}$ applied field will be enhanced by the ferromagnetic nature of the film. The remnant magnetization of the nickel film produces a significant magnetic field in the oxygen film layer when the external field is applied and the estimated field experience by the adlayers is the magnetic induction and remnant magnetization for the $30 \mathrm{~nm}$ nickel sample are estimated to be around $340 \mathrm{G}$ and $60 \mathrm{G}$, respectively, for the applied field of $35 \mathrm{G}$. [51-53]. These are the values for within the nickel film. At the surface they are further enhanced by $11 \%$ [53]. The resultant field strength decreases with distance away from the surface, in accordance to $1 / \mathrm{r}^{3}$ law [56,57]. Overall, therefore, an external field of $70 \mathrm{G}$ applied to the gold substrate has a comparable impact on the oxygen layer as that of a $35 \mathrm{G}$ external field applied to a nickel surface [51,52].

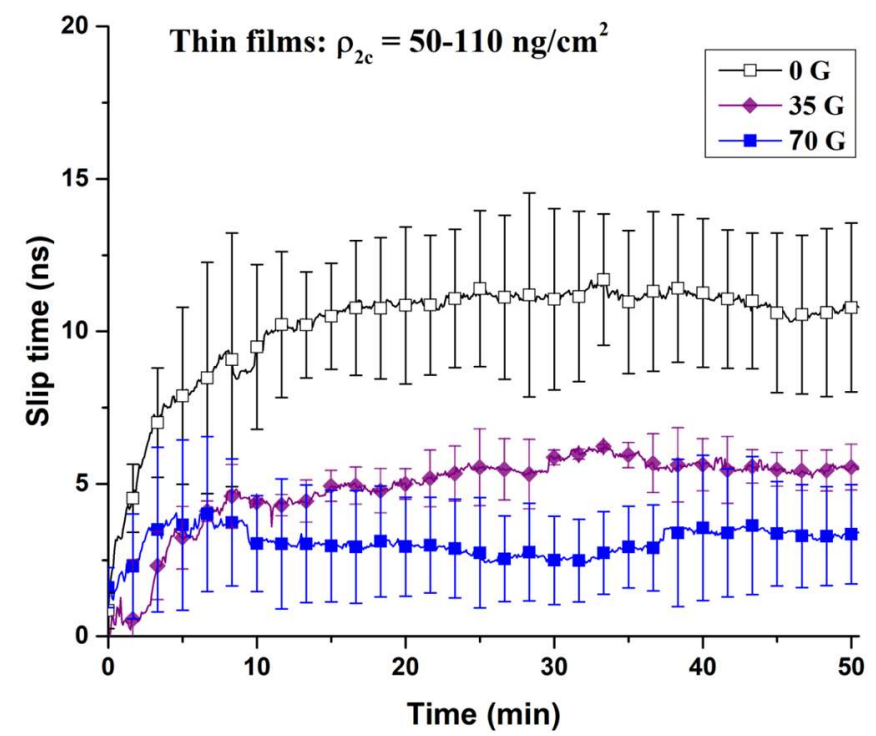

Figure 5. Average slip time versus time for low coverage oxygen films on gold at $47.5 \mathrm{~K}$ in the presence and absence of an external magnetic field perpendicular to the surfaces. Error bars represent the standard deviation of the slip times measured over multiple measurements at fixed points in time. Gas is admitted to the chamber at time $\mathrm{t}=0 \mathrm{~min}$. The effective field on the oxygen film in the external field of $70 \mathrm{G}$ for Au QCM surface is comparable to that of $35 \mathrm{G}$ external field for Ni QCM surface [51,52]. Slip times are observed to be shorter in the presence of an external magnetic field of $35 \mathrm{G}$, indicating higher friction levels.

The most notable feature of the slip time data in Figure 5 for thin films on gold is the opposite nature of the trends induced by the presence of an applied field. In zero fields, the slip time for gold is approximately $10 \mathrm{~ns}$, and for nickel is it $20 \mathrm{~ns}$, a difference that is readily attributable to differences in the chemical nature of the substrates. In the presence of an applied field, the friction is observed to increase substantially for the gold substrates while dropping somewhat for the nickel substrates. The various contributions to friction will be discussed in the next section.

\section{Analysis of Friction Levels from Various Contribution Mechanisms.}

Three phenomena will be considered in this section within the context of the experimental observations: (1) Magnetostrictive effects, whereby field induced changes to the physical structure of the molecules gives rise secondary effects that impact friction levels; (2) field-induced changes to the magnetic spins of the adsorbate and substrate that give rise to intrinsic magnetic friction; and (3) contributions from eddy currents. 


\subsection{Magnetostriction and Magnetic Spin Effects}

Magnetostriction, a structural reorientation of elements of magnetic material in an applied magnetic field, will result in changes to friction via changes in interfacial commensurability. It is likely to occur with both the gold and nickel substrates, as the measurements are performed at temperatures close to where structural transitions would occur even in the absence of an applied field. It is known that the liquid, $\theta$, and fluid phases of $2 \mathrm{D}$ adsorbed $\mathrm{O}_{2}$ have molecular alignment which is freely rotating, but prefers to tilt in the in-plane direction. Near the boundary with the $\zeta$ phase (molecules standing) a perpendicular magnetic field grants the molecule energy if it aligns. The data taken on thin $\mathrm{O}_{2}$ films on $\mathrm{Ni}$ at $47.5 \mathrm{~K}$ exist in the fluid phase near this boundary with the $\zeta$ phase, which is shown [58] to have phase boundaries in this region. The $\delta$ to $\zeta$ transition in particular is considered to be driven by magnetic energies, and magnetic energies account for 30-100 K per molecule.

It is likely that the phase diagram for $\mathrm{O}_{2}$ physically adsorbed on gold, $\mathrm{Ni}$ and $\mathrm{NiO}$ exhibits a similar boundary structure to the diagram for $\mathrm{O}_{2}$ on graphene. An analogous molecular reorientation transition into perpendicular direction occurs for increased coverage at $20 \mathrm{~K}$. The location of phase boundaries for $\mathrm{O}_{2}$ on $\mathrm{Ni}$ are likely to be shifted in relative position, since adsorption energies for $\mathrm{O}_{2}$ on $\mathrm{Ni}$ are larger than those values known for $\mathrm{O}_{2}$ on $\mathrm{Gr}$. This implies the $\mathrm{O}_{2}$ on $\mathrm{Ni}$ phase structure is shifted to the right because adsorbates are more energetically encouraged to adsorb on $\mathrm{Ni}$ at a given temperature than they would be on graphene. It is not known whether the analogous fluid, theta, and epsilon phases exist for adsorption on $\mathrm{Ni}$, although it is plausible given $\mathrm{Ni}$ interatomic spacing of $0.249 \mathrm{~nm}$ similar to $0.142 \mathrm{~nm}$ graphene nearest neighbor spacing in the hexagonal lattice. Graphene's hexagonal lattice structure has been shown to influence the differences in epitaxy for the theta, fluid, and liquid phases; this situation is different with the triangular lattice of $\mathrm{Ni}(111)$ for example.

The energy difference equivalent to $1 \mathrm{~K}$ cooling is $1 \mathrm{k}_{\mathrm{B}}=9 \times 10^{-5} \mathrm{eV}$. How does the energy introduced by the external field compare to an equivalent $1 \mathrm{~K}$ cooling in the phase structure? When the external field is at a strength of 35 gauss, the magnetic field in the vicinity of $\mathrm{Ni}$ is 360 gauss due to the magnetization field of Ni. The Zeeman energy associated with an oxygen molecular spin moment orienting in field is:

$$
E_{z}=3.6 \times 10^{-2} \times 2 \mu_{B}=4.2 \times 10^{-6} \mathrm{eV}
$$

which is about $5 \%$ of the equivalent thermal energy lost by cooling $1 \mathrm{~K}$. In the region of the phase diagram close to a transition, it is possible for a field to bring the system across the transition solely due to Zeeman energy supplied by the field. Our data on $\mathrm{O}_{2}$ on $\mathrm{Ni}$ films take place at $47 \mathrm{~K}$ and are lying within $5 \mathrm{~K}$ of the transition to $\zeta$. This transition is known to be broad, showing coexistence of both phases within $0.5 \mathrm{~K}$ of the boundary.

For an oxygen molecule existing as member of a film, magnetic exchange also plays a role in the total magnetic energy of the $\mathrm{O}_{2}$ molecule. Magnetic energies for the oxygen intermolecular interaction are estimated to be $40 \mathrm{~K}[59]$ at $3.2 \AA$, with an exponential dependence on distance, whereas average vdW adsorption energy on graphite is $1122 \mathrm{~K}$ [60]. Due to the magnetic exchange between oxygen molecules is non-negligible for the condensed phases, behavior in an external field will be much more pronounced than just that expected by Zeeman splitting (which is typically the valid treatment for non-interacting moments). The bulk $\gamma$ oxygen phase and bulk liquid are known to have short-ranged antiferromagnetic order. For antiferromagnetism, the exchange constant is negative. For this reason, if the $\zeta$ phase is ordered antiferromagnetically, due to its (approximately) triangular lattice, it is likely to be magnetically frustrated.

We now consider also the situation of the field application causing the molecular magnetic moments to align while the physical structure of the molecule stays unaligned. The spin-spin energy of an oxygen molecule is $0.13 \mathrm{meV}$ in preference for the interatomic axis as compared to lying in the plane perpendicular to the molecular axis [61]. Since this energetic preference is larger by a factor of 30 than the external field-dipole field energy, we can consider the spin moment of $\mathrm{O}_{2}$ to be rigidly attached to its axis, meaning a change in the magnetic moment direction causes a concomitant 
change in the physical orientation of the molecule. In some low temperature phases of adsorbed oxygen, the magnetic moments point in a direction other than the molecular axis, in these situations a vdW-exchange competition takes place.

Magnetic thin films exhibit magnetization reorientation in removal of field due to shape anisotropy. If this phenomenon is present in our films, it implies that the field absent condition causes the remnant field in the nickel film to reorient in an in-plane direction, perhaps causing the adsorbate molecules follow suit and lay flat, further encouraging them to mimic the $\delta$ phase. This expectation underlies our interpretation of the incremental warmup data of $\mathrm{O}_{2}$ on $\mathrm{Ni}$. The increased frictional levels observed in our data upon lowering of the system temperature from $47 \mathrm{~K}$ through $30 \mathrm{~K}$ for film coverages in the $\rho=50 \mathrm{ng} / \mathrm{cm}^{2}$ region implies the films have undergone a transition from fluid to $\delta+\zeta$ phase, here preferring the $\delta$ phase due to the in-plane remnant field $\mathrm{M}=60 \mathrm{~g}$ expected to be present near the nickel.

\subsection{Impact of Molecular Orientation on Friction Levels.}

To assess the impact of the physical orientation of the molecule upon its frictional interaction with the substrate, we examine the energy corrugation amplitude framework. A given substrate has lateral modulations in the van der Walls energy of an adsorbate molecule attached to the surface. The slip time of an adsorbed layer due to phonon friction depends on the adsorbate-substrate corrugation amplitude [2,4,62]:

$$
\tau \propto f^{-2}
$$

where $\tau$ is slip time and $\mathrm{f}$ is corrugation amplitude. In order to account for a factor of 1.8 increase in slip time when field is applied:

$$
\frac{\tau_{B}}{\tau_{0}}=\frac{f_{0}^{2}}{f_{B}^{2}}=1.8
$$

where $\tau_{B}$ is slip time in field, $\tau_{0}$ is slip time in absence of field, $f_{B}$ is amplitude in field and $f_{0}$ is amplitude in absence of field. The van der Waals potential between two particles follows a 6-12 form, which is dominated by the $1 / r^{6}$ dependence beyond the adsorption distance, which is the realm we are considering:

$$
U=E_{L J} \propto \frac{1}{r^{6}}
$$

Integrating this interaction over the surface yields an interaction law:

$$
U(r) \propto \frac{1}{r^{3}}
$$

which implies that for differences in adsorbate energies:

$$
f=\left|U_{\text {atom }}-U_{\text {site }}\right| \Rightarrow f \propto \frac{1}{r^{3}}
$$

Inserting this into the above dependence:

$$
\frac{\tau_{B}}{\tau_{0}}=\frac{r_{B}^{6}}{r_{0}^{6}}
$$

Our equilibrium adsorption distance for $\mathrm{O}_{2}, 3.2 \AA$ [61] yields an effective field adsorption distance:

$$
r_{B}=\sqrt[6]{1.8 * 3.2^{6}}=3.5 \AA
$$

This implies that when the molecule stands upright, its effective adsorption amplitude is lessened by an amount corresponding to a displacement by:

$$
\delta r=r_{B}-r_{0}=3.5-3.2 \AA=0.3 \AA
$$


If the ellipsoidal shape of the $\mathrm{O}_{2}$ is taken into account, the short axis radius is $1.6 \AA$ and the long axis radius is $2.1 \AA$, and the difference between these is $0.5 \AA$. By accounting for shape anisotropy, the results are consistent with the explanation of the frictional behavior in a field.

A freely rotating $\mathrm{O}_{2}$ molecule on graphene has energy difference $1149 \mathrm{~K}-1112 \mathrm{~K}=37 \mathrm{~K}=$ 3.2 MeV, which we refer to as corrugation amplitude, between site and atom adsorption positions. For $\mathrm{O}_{2}$ on $\mathrm{Ni}$ or $\mathrm{NiO}$ the corrugation energy amplitude is assumed to be larger, because graphene is known to be relatively 'flat' in terms of van der Waals adsorption as compared to most substrates. The above treatment is in fact independent of corrugation amplitude value. The shift in effective adsorption distance is solely dependent on original adsorption distance, which for $\mathrm{O}_{2}$ on $\mathrm{NiO}$ is expected to be smaller than for $\mathrm{O}_{2}$ on graphene. Other subtleties exist; the oxygen molecule has a polarizability anisotropy $\mathrm{k}$ which alters the van der Walls binding dependent on direction. As well, the corrugation frequency is changed [62]. For the purposes of phononic friction, the polarizability is the same for molecular orientation parallel or anti-parallel, since for both of these alignments the molecule is standing perpendicular to the substrate. Thus, this treatment works for antiferromagnetic state as if it were ferromagnetic.

This mechanism of magnetostriction could account for the decrease in friction for the thin $\mathrm{O}_{2}$ film on $\mathrm{Ni}$ in applied field, and predicts a frictional increase upon transition from the fluid to the delta phase, for which the films are flat, and therefore closer to the surface.

\subsection{Eddy Current and Conduction Electron Effects.}

The increase in friction for oxygen layers on gold substrates cannot be accounted for by structural reordering. Such effects are likely to be present, however, a competing and dominant effect is apparently also present which gives rise to the opposing trends for films on the gold substrates. The most likely mechanism is the formation of eddy currents, which are expected to be stronger for the gold substrates given the major differences (skin depth, surface conductivity, etc.), between gold, nickel, and nickel films with surface oxide layers [63]. The contributions of conduction electron friction predicted analytically by Bruch [5] are of the same order as that observed here. Eddy currents and/or conduction electron dissipations mechanisms therefore are likely candidates for the observed behaviors.

Further modeling efforts are clearly required to reveal the underlying mechanisms for the observed phenomena. In order to perform a complete study, a combination of MC and MD simulations, in combination with DFT are required. DFT allows an estimate of the exchange interactions and possible modifications to intermolecular potentials, and also provides an estimate of the electron density to determine preferred bonding sites. MC techniques estimate spin exchange couplings between the constituent molecules, while MD can be employed to determine the film structure, orientation and sliding friction levels.

\section{Summary and Conclusions}

We have reported here a study of the sliding friction levels of oxygen monolayer and multilayer films adsorbed on nickel and gold substrates close to the oxygen monolayer solid-liquid melting transition temperature in the presence and absence of a weak external magnetic field. Friction levels for the monolayers in the presence of the field were observed to be half of those observed in the absence of a field, and the data are consistent with interfacial effects as the source of the magnetic sensitivity. Friction levels for thin films on gold were meanwhile observed to be substantially increased in the presence of the field. Magnetically-induced structural reorientation (magnetostriction) and/or realignment of adlayer spins, both of which would reduce structural and/or magnetic interfacial corrugation and corrugation, potentially underlie the observed reductions in friction levels. Conduction electron effects fail outright to explain the data for the nickel data but are viable candidates for the gold data. Pressure induced collapse of friction in response to the increased normal force is improbable, but not strictly ruled out. 
Although the scope of the present study is limited in nature, our work demonstrates a first step in documenting the role of magnetic effects in a system that is readily accessible to both experimental and theoretical investigations of such mechanisms. The ability to apply an external field to "tune" the friction moreover allows active control of friction, going beyond the usual passive observations of frictional energy phenomena. A variety of future experimental and theoretical studies have been suggested that will illuminate the various contributions of intrinsically magnetic dissipation mechanisms as well as a variety of secondary effects that accompany them.

Author Contributions: Z.B.F. and J.K. conceived and designed the experiments; Z.B.F., K.M.F., S.G.K., and B.A. performed the experiments; Z.B.F., B.A., and J.K. analyzed the data; Z.B.F. wrote the initial draft and all authors contributed in editing/revising the manuscript.

Acknowledgments: Support from NSF DMR1310456 (JK) is gratefully acknowledged. Discussions with D.B. Dougherty, D.E. Aspnes, and M. Hodak are greatly appreciated.

Conflicts of Interest: The authors declare no conflict of interest.

\section{References}

1. Holmberg, K.; Andersson, P.; Erdemir, A. Global energy consumption due to friction in passenger cars. Tribol. Int. 2012, 47, 221-234. [CrossRef]

2. Krim, J. Friction and energy dissipation mechanisms in adsorbed molecules and molecularly thin films. Adv. Phys. 2012, 61, 155-323. [CrossRef]

3. Park, J.Y.; Salmeron, M. Fundamental aspects of energy dissipation in friction. Chem. Rev. 2014, 114, 677-711. [CrossRef] [PubMed]

4. Robbins, M.O.; Krim, J. Energy Dissipation in Interfacial Friction. MRS Bull. 1998, 23, 23-26. [CrossRef]

5. Bruch, L. Ohmic damping of center-of-mass oscillations of a molecular monolayer. Phys. Rev. B 2000, 61, 16201-16206. [CrossRef]

6. Highland, M.; Krim, J. Superconductivity dependent friction of water, nitrogen, and superheated he films adsorbed on Pb(111). Phys. Rev. Lett. 2006, 96, 226107. [CrossRef]

7. Persson, B.N.J. Surface resistivity and vibrational damping in adsorbed layers. Phys. Rev. B 1991, 44, 3277-3296. [CrossRef]

8. Park, J.Y.; Ogletree, D.F.; Thiel, P.A.; Salmeron, M. Electronic Control of Friciton in Silicon pn junctions. Science 2006, 313, 5784. [CrossRef]

9. Yao, L.; Guo, W. Spin friction in two-dimensional antiferromagnetic crystals. Phys. Rev. B 2018, 97, 104302.

10. Gzik-Szumiata, M.; Szumiata, T.; Morozow, D. Modelling of Spin-Dependent Mechanical Friction at Atomic Level. Acta Phys. Pol. A 2018, 133, 713-715. [CrossRef]

11. Kadau, D.; Hucht, A.; Wolf, D.E. Magnetic friction in Ising spin systems. Phys. Rev. Lett. 2008, 101, 13. [CrossRef]

12. Wolter, B.; Yoshida, Y.; Kubetzka, A.; Hla, S.W.; Von Bergmann, K.; Wiesendanger, R. Spin friction observed on the atomic scale. Phys. Rev. Lett. 2012, 109, 11. [CrossRef] [PubMed]

13. Lehtinen, P.O.; Foster, A.S.; Ayuela, A.; Krasheninnikov, A.; Nordlund, K.; Nieminen, R.M. Magnetic Properties and Diffusion of Adatoms on a Graphene Sheet. Phys. Rev. Lett. 2003, 91, 17202. [CrossRef] [PubMed]

14. Valencia, A.M.; Caldas, M.J. Single vacancy defect in graphene: Insights into its magnetic properties from theoretical modeling. Phys. Rev. B 2017, 96, 125431. [CrossRef]

15. Ouazi, S.; Kubetzka, A.; von Bergmann, K.; Wiesendanger, R. Enhanced Atomic-Scale Spin Contrast due to Spin Friction. Phys. Rev. Lett. 2014, 112, 076102. [CrossRef]

16. Weinberger, B.R.; Lynds, L.; Hull, J.R.; Balachandran, U. Low friction in high temperature superconductor bearings. Appl. Phys. Lett. 1991, 59, 1132-1134. [CrossRef]

17. Magiera, M.P.; Brendel, L.; Wolf, D.E.; Nowak, U. Spin waves cause non-linear friction. Europhys. Lett. 2011, 95, 17010. [CrossRef]

18. Fusco, C.; Wolf, D.E.; Nowak, U. Magnetic friction of a nanometer-sized tip scanning a magnetic surface: Dynamics of a classical spin system with direct exchange and dipolar interactions between the spins. Phys. Rev. B 2008, 77, 174426. [CrossRef] 
19. She, J.H.; Balatsky, A.V. Noncontact friction and relaxational dynamics of surface defects. Phys. Rev. Lett. 2012, 108, 136101. [CrossRef]

20. Hoffmann, B.; Houbertz, R.; Hartmann, U. Eddy current microscopy. Appl. Phys. 1998, 66, 409-413. [CrossRef]

21. Gabureac, M.; Viret, M.; Ott, F.; Fermon, C. Magnetoresistance in nanocontacts induced by magnetostrictive effects. Phys. Rev. B 2004, 69, 2-5. [CrossRef]

22. Nielsen, M.; McTague, J.P. Oxygen monolayers adsorbed on graphite studied by neutron scattering. Phys. Rev. B 1979, 19, 3096-3106. [CrossRef]

23. Barylnik, A.S.; Prokhvatilov, A.I. Spontaneous magnetostriction effects in alpha-phases and beta-phases of oxygen. Fiz. Nizk. Temp. 1994, 20, 912-917.

24. Chen, C.Y.; Hong, C.Y.; Wang, S.W. Magnetic flows in a tube with the effects of viscosity variation. J. Magn. Magn. Mater. 2002, 252, 253-255. [CrossRef]

25. Murakami, Y.; Suematsu, H. Magnetic and melting transitions of oxygen monolayers and multilayers physisorped on exfoliated graphite. Phys. Rev. B 1996, 54, 4146-4154. [CrossRef]

26. Altman, I.S.; Pikhitsa, P.V.; Kim, Y.-J.; Choi, M. Magnetism of adsorbed oxygen at low coverage. Phys. Rev. B 2003, 67, 144410. [CrossRef]

27. Marx, R.; Christoffer, B. Magnetocaloric effects of 2D adsorbed O2. J. Phys. C 1985, 18, 2849-2858. [CrossRef]

28. Bhethanabotla, V.R.; Steele, W.A. Computer-simulation study of melting in dense oxygen layers on graphite. Phys. Rev. B 1990, 41, 9480-9487. [CrossRef]

29. Krim, J.; Coulomb, J.P.; Bouzidi, J. Triple-point wetting and surface melting of oxygen films adsorbed on graphite. Phys. Rev. Lett. 1987, 58, 583-586. [CrossRef] [PubMed]

30. Chiarello, R.; Coulomb, J.P.; Krim, J.; Wang, C.L. Surface melting of multilayer oxygen films on graphite studied by neutron diffraction. Phys. Rev. B 1988, 38, 8967-8973. [CrossRef]

31. Bhethanabotla, V.; Steele, W. Simulations of O2 adsorbed on graphite at $45 \mathrm{~K}$ : The monolayer to bilayer transition. Can. J. Chem. 1988, 66, 866-874. [CrossRef]

32. Ulbricht, H.; Moos, G.; Hertel, T. Physisorption of molecular oxygen on single-wall carbon nanotube bundles and graphite. Phys. Rev. B 2002, 66, 75404. [CrossRef]

33. Aoki, M.; Taoka, H.; Kamada, T.; Masuda, S. Local electronic states of oxygen on a Ni(111) surface studied by metastable atom electron spectroscopy. J. Electron Spectrosc. Relat. Phenom. 2001, 114-116, 507-512. [CrossRef]

34. Yamagishi, S.; Jenkins, S.J.; King, D.A. First principles studies of chemisorbed O on Ni $\{111\}$. Surf. Sci. 2003, 543, 12-18. [CrossRef]

35. Kazama, Y.; Matsumoto, M.; Sugimoto, T.; Okano, T.; Fukutani, K. Low-temperature surface phase and phase transition of physisorbed oxygen on the Ag(111) surface. Phys. Rev. B 2011, 84, 064128. [CrossRef]

36. Kurahashi, M.; Sun, X.; Yamauchi, Y. Magnetic properties of $\mathrm{O}_{2}$ adsorbed on $\mathrm{Cu}(100)$ : A spin polarized metastable He beam study. Phys. Rev. B 2012, 86, 245421. [CrossRef]

37. Gnecco, E.; Meyer, E. (Eds.) Fundamentals of Friction and Wear on the Nanoscale; Springer International Publishing: Cham, Switzerland, 2015.

38. Daly, C.; Krim, J. Sliding Friction of Solid Xenon Monolayers and Bilayers on Ag(111). Phys. Rev. Lett. 1996, 76, 803-806. [CrossRef]

39. Johannsmann, D. Viscoelastic, mechanical, and dielectric measurements on complex samples with the quartz crystal microbalance. Phys. Chem. Chem. Phys. 2008, 10, 4516. [CrossRef]

40. Krim, J.; Widom, A. Damping of a crystal oscillator by an adsorbed monolayer and its relation to interfacial viscosity. Phys. Rev. B 1988, 38, 12184-12189. [CrossRef]

41. Watts, E.T.; Krim, J.; Widom, A. Experimental observation of interfacial slippage at the boundary of molecularly thin films with gold substrates. Phys. Rev. B 1990, 41, 3466-3472. [CrossRef]

42. Sokoloff, J.B.; Krim, J.; Widom, A. Determination of an atomic-scale frictional force law through quartz-crystal microbalance measurements. Phys. Rev. B 1993, 48, 9134-9137. [CrossRef]

43. Hanke, S.; Petri, J.; Johannsmann, D. Partial slip in mesoscale contacts: Dependence on contact size. Phys. Rev. E 2013, 88, 32408. [CrossRef] [PubMed]

44. Smith, E.D.; Robbins, M.O.; Cieplak, M. Friction on adsorbed monolayers. Phys. Rev. B 1996, 54, 8252-8260. [CrossRef]

45. Tomassone, M.S.; Sokoloff, J.B.; Widom, A.; Krim, J. Dominance of Phonon Friction for a Xenon Film on a Silver(111) Surface. Phys. Rev. Lett. 1997, 79, 4798-4801. [CrossRef] 
46. Franchini, A.; Bortolani, V.; Santoro, G.; Brigazzi, M. Sliding friction of N 2 on Pb(111). J. Phys. Condens. Matter 2010, 22, 304002. [CrossRef]

47. Mak, C.; Krim, J. Quartz-crystal microbalance studies of the velocity dependence of interfacial friction. Phys. Rev. B 1998, 58, 5157-5159. [CrossRef]

48. Bruschi, L.; Carlin, A.; Mistura, G. Depinning of Atomically Thin Kr Films on Gold. Phys. Rev. Lett. 2002, 88, 46105. [CrossRef]

49. Reviakine, I.; Morozov, A.N.; Rossetti, F.F. Effects of finite crystal size in the quartz crystal microbalance with dissipation measurement system: Implications for data analysis. J. Appl. Phys. 2004, 95, 7712-7716. [CrossRef]

50. Cassiède, M.; Paillol, J.H.; Pauly, J.; Daridon, J.-L. Electrical behaviour of AT-cut quartz crystal resonators as a function of overtone number. Sens. Actuators A Phys. 2010, 159, 174-183. [CrossRef]

51. Edwards, R.L. The magnetic properties of evaporated nickel and iron films. Phys. Rev. 1927, 29, 321-331. [CrossRef]

52. Sorensen, A.J. Magnetic Properties of Thin Films of Ferromagnetic Metals Produced by the Evaporation Method. Phys. Rev. 1924, 24, 658-665. [CrossRef]

53. Mittendorfer, F.; Eichler, A.; Hafner, J. Structural, electronic and magnetic properties of nickel surfaces. Surf. Sci. 1999, 423, 1-11. [CrossRef]

54. Bruschi, L.; Fois, G.; Pontarollo, A.; Mistura, G.; Torre, B.; de Mongeot, F.B.; Boragno, C.; Buzio, R.; Valbusa, U. Structural depinning of Ne monolayers on $\mathrm{Pb}$ at $\mathrm{T}<6.5$ K. Phys. Rev. Lett. 2006, 96, 216101. [PubMed]

55. Bruschi, L.; Pierno, M.; Fois, G.; Ancilotto, F.; Mistura, G.; Boragno, C.; de Mongeot, F.B.; Valbusa, U. Friction reduction of Ne monolayers on preplated metal surfaces. Phys. Rev. B 2010, 81, 115419. [CrossRef]

56. Jackson, J.D. Classical Electrodynamics, 3rd ed.; John Wiley \& Sons: Hoboken, NJ, USA, 2007.

57. Blundell, S.; Thouless, D. Magnetism in Condensed Matter. Am. J. Phys. 2003, 71, 94. [CrossRef]

58. Toney, M.F.; Fain, S.C. Low-energy electron diffraction determination of the structure of the phase of oxygen physisorbed on graphite. Phys. Rev. B 1984, 30, 1115-1118. [CrossRef]

59. Freiman, Y.A.; Jodl, H.J. Solid oxygen. Phys. Rep. 2004, 401, 1-4. [CrossRef]

60. Toney, M.F.; Fain, S.C. Low-energy electron diffraction study of molecular oxygen physisorbed on graphite. Phys. Rev. B 1987, 36, 1248-1258. [CrossRef]

61. Herzberg, G. Molecular Spectra and Molecular Structure I. Diatomic Molecules; Prentice Hall: New York, NY, USA, 1966.

62. Coffey, T.; Krim, J. Impact of substrate corrugation on the sliding friction levels of adsorbed films. Phys. Rev. Lett. 2005, 95, 076101. [CrossRef] [PubMed]

63. Rose, J.H.; Tai, C.C.; Moulder, J.C. Extreme sensitivity of eddy-currents to the surface conditions of nickel. In Review of Progress in Quantitative Nondestructive Evaluation; Springer: Boston, MA, USA, 1997; pp. 249-255. 